\title{
On the Transition from a Biomimetic Molecular Switch
}

\section{to a Rotary Molecular Motor}

Marco Paolino,,${ }^{\mathrm{a},}$ Tommaso Giovannini, ${ }^{\mathrm{b}}$ Madushanka Manathunga, ${ }^{\mathrm{c}}$ Loredana Latterini, ${ }^{\mathrm{d}}$ Giulia

Zampini, ${ }^{\mathrm{d}}$ Robin Pierron, ${ }^{\mathrm{e}}$ Jérémie Léonard, ${ }^{\mathrm{e}}$ Stefania Fusi, ${ }^{\mathrm{a}}$ Gianluca Giorgi, ${ }^{\mathrm{a}}$ Germano Giuliani, ${ }^{\mathrm{a}}$ Andrea Cappelli, ${ }^{\mathrm{a}}$ Chiara Cappelli, ${ }^{\mathrm{b}}$ Massimo Olivucci ${ }^{\mathrm{a}, \mathrm{c},},{ }^{*}$

a Dipartimento di Biotecnologie, Chimica e Farmacia, Università di Siena, Via A. Moro 2, 53100 Siena, Italy;

${ }^{\text {b }}$ Scuola Normale Superiore, Piazza dei Cavalieri 7, 56126 Pisa, Italy

${ }^{\mathrm{c}}$ Chemistry Department, Bowling Green State University, USA;

${ }^{\mathrm{d}}$ Dipartimento di Chimica, Biologia e Biotecnologie, Università di Perugia, Via Elce di Sotto, 8, 06123 Perugia, Italy

${ }^{\text {e }}$ Université de Strasbourg, CNRS, Institut de Physique et Chimie des Matériaux de Strasbourg, UMR 7504, F-67000 Strasbourg, France

E-mail: paomar@oneonline.it, molivuc@bgsu.edu 


\begin{abstract}
The experimental investigation of the unidirectional motion characterizing the photoisomerization of single-molecule rotary motors, requires accessible lab prototypes featuring an ECD signal sensitive to the geometrical and electronic changes occurring during an ultrafast reactive process. Here we report a combined experimental/computational study of a candidate obtained via the asymmetrization of a light-driven biomimetic molecular switch. We show that the achieved motor has an ECD band remarkably sensitive to the isomerization motion and it is therefore suitable for time-resolved ECD studies. However, we also find that, unexpectedly, the synthesized motor isomerizes on a time-scale longer than the sub-picosecond time measured for the achiral parent. A result that points to alternative candidates conserving a high reaction speed.
\end{abstract}

\title{
TOC Graphic
}
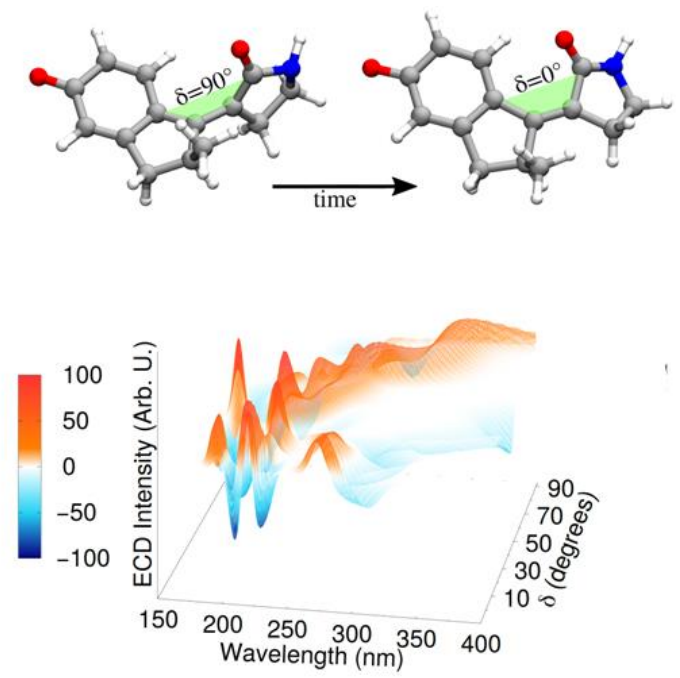
Natural molecular motors are nanometer macromolecular assemblies capable of converting chemical energy in mechanical motion. ${ }^{1-3}$ These biological "devices" inspired the design and synthesis of subnanometer molecular motors expected to play a pivotal role in the development of artificial nanodevices ${ }^{4-8}$ by converting chemical or light energy in directional motion of specific molecular or supramolecular parts. ${ }^{9,10}$ In the present work, we focus on single-molecule rotary motors fuelled by light-driven isomerizations of an asymmetric (i.e. chiral) olefin framework. ${ }^{11}$ More specifically, these systems are capable of achieving the full rotation of a moiety called "rotor" with respect to a fixed "stator" via consecutive $Z$-to- $E$ and $E$-to- $Z$ unidirectional (i.e. either clockwise or counterclockwise) photoisomerization of a specific $\mathrm{C}=\mathrm{C}$ double bond separated by conformational helix-inversion steps. ${ }^{6}$

In the past, researchers have been looking for ways of designing fast rotary motors and measuring the rotational direction and speed of the rotor. ${ }^{12-15}$ Fast rotors are needed because, while a slow motor has the advantage of allowing a study of the rotation via stationary spectroscopies, only a high rotational speed is suitable for applications requiring synchronous rotations at the population level. ${ }^{16-}$ ${ }^{20}$ To achieve that thermally randomized motion have to be avoided by designing motors with low or absent thermal helix-inversion barriers, but also sub-picosecond $\mathrm{C}=\mathrm{C}$ isomerizations are sought after, with the expectation that they may enable a level of control on the rotational directionality and photoisomerization quantum yield similar to what observed in the prototypical example of visual pigments. ? Due to the chirality of rotary motors, time-resolved electronic circular dichroism (ECD) measurements would, in principle, allow to follow a sub-picosecond directional $\mathrm{C}=\mathrm{C}$ twist. Such measurements represent a timely target as, in recent years, ECD has been implemented in pumpprobe experiments allowing to track the variations of UV-vis circular dichroism signals on the subpicosecond time-scale. ${ }^{22-24}$ In addition, pump-probe vibrational circular dichroism (VCD) has been developed in the mid-IR spectral range to monitor the structural changes on the picosecond timescale. ${ }^{25,26}$ 
Over the past 10 years, we have been involved in the design, synthesis and characterization of lightdriven molecular switches (LDMSs), i.e. achiral systems that do not rotate unidirectionally. More specifically, we have used a biomimetic strategy to prepare positively charged N-alkylated or Nprotonated indanylidene-pyrroline Schiff bases (NAIPs and NHIPs respectively) ${ }^{27-33}$ that mimic the sub-picosecond $\mathrm{C}=\mathrm{C}$ isomerization of the retinal chromophore of visual pigments. ${ }^{34-36} \mathrm{~A}$ recent development led to chiral NAIP derivative bearing a stereogenic center on the indene stator (Fig. 1, left) and, thus, capable of biasing a cationic pyrroline rotor to rotate unidirectionally about the molecule exocyclic $\mathrm{C}=\mathrm{C}$ bonds ${ }^{37,}{ }^{38}$ Although the resulting $\mathrm{Ch}-\mathrm{dMe}-\mathrm{MeO}-\mathrm{NAIP}$ system constitutes, in principle, a light-driven molecular motor (LDMMs), it shows a too-weak ECD signal not suitable for experimental studies. ${ }^{37}$
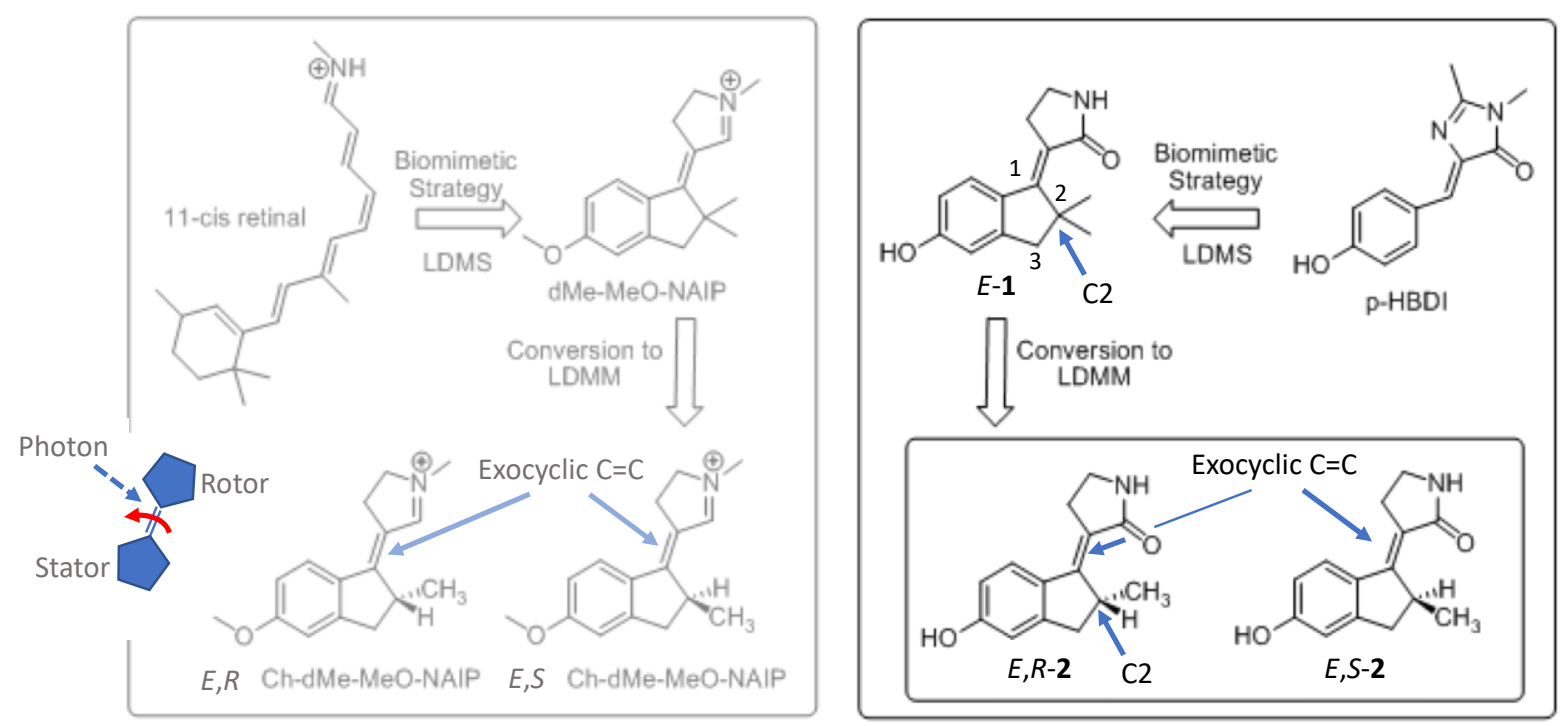

Figure 1. From LDMSs to chiral LDMMs. LDMMs miming the Rhodopsin chromophore (left) and the GFP fluorophore (right), displayed in their $E$ diastereoisomer. A schematic representation of a single-molecule rotary motor is shown on the left.

With the hope to achieve the preparation of a sub-picosecond rotating LDMM with a strong ECD signal, we present complementary experimental and computational studies of a chiral derivative of the biomimetic light-driven LDMS 1 mimicking the para-hydroxybenzylidenedimethylimidazolone (p-HBDI, Fig 1 right) fluorophore of the Green Fluorescent Protein (GFP) and known to display a sub-picosecond photoisomerization of both its $Z$ and $E$ diastereomers. ${ }^{38}$ More specifically, we report 
on the design, synthesis and characterization of the LDMM 2 in racemic form, produced from $\mathbf{1}$ by inserting a stereogenic center in its stator to bias the rotation direction about the isomerizing $\mathrm{C}=\mathrm{C}$ bond. By combining steady-state and transient UV-Vis spectroscopies with computational studies carried out using two different levels of theory, we show that $\mathbf{2}$ represents a suitable lab model for future time-resolved ECD studies of the unidirectional rotary motion. The results are presented and discussed below where we sequentially deal with preparation, spectroscopic characterization and computational studies of equilibrium and non-equilibrium structures. Whenever possible the properties of $\mathbf{2}$ are compared with those reported for the homologue achiral compound $1 .^{38}$

Synthesis. Promotion to the first singlet excited state $\left(S_{1}\right)$ of the anionic form of $\mathbf{1}$ triggers a charge translocation from the phenolic oxygen of the indene moiety (the stator) towards the pyrrolidone head (the rotor) unlocking the exocyclic $\mathrm{C}=\mathrm{C}$ bond that isomerizes on the desired sub-picosecond timescale. ${ }^{38}$ Thus, in order to turn the LDMS 1 into a LDMM we decided to include a stereogenic center, expected to bias the rotation direction without changing the electronic structure of the molecule. As shown in Fig. 1, this has been accomplished by "removing" one of the two C2 methyl substituents of 1.

\section{Scheme 1.}




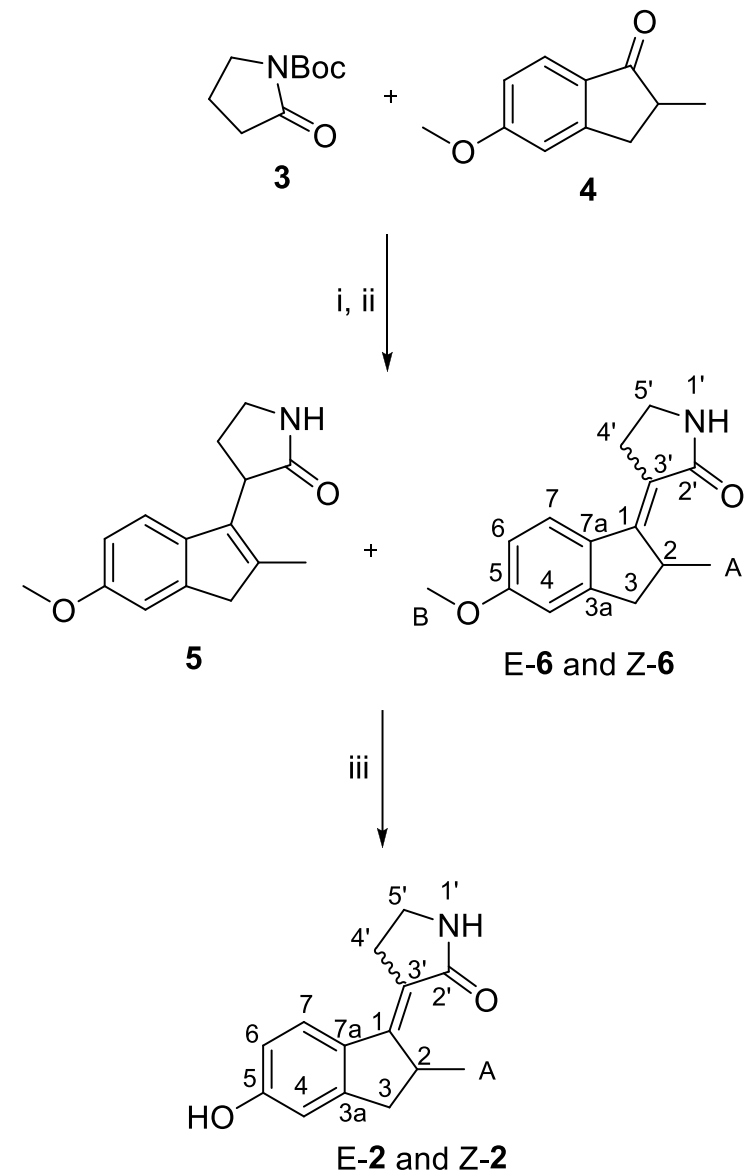

Reagents: (i) N-Boc-pyrrolidinone, $\mathrm{LiHDMS}_{3} \mathrm{BF}_{3}(\mathrm{Et})_{2} \mathrm{O}$, THF; (ii) TFA, $\mathrm{CH}_{2} \mathrm{Cl}_{2}$; (iii) $\mathrm{BBr}_{3}, \mathrm{CH}_{2} \mathrm{Cl}_{2}$.

Compound 2 was synthesized starting from the available 5-methoxy-2-methyl-1-indanone using the strategy shown in Scheme 1. The racemic mixture of $\mathbf{4}$ was reacted with N-Boc-pyrrolidinone (3) in order to obtain the $E$ and $Z$ isomers of 6 (yield $47 \%, E / Z$ ratio 8:2) by dehydration of the aldol condensation intermediate with TFA. The presence of only one methyl group in C2 (see Scheme 1 for the numbering) of the 5-methoxy-2-methyl-1-indanone led to the formation of the undesired compound 5 (yield 47\%) due to the competition between the endo and exocyclic dehydration of the intermediate alcohol. However, the deprotection of the methoxy group in both compounds $\mathbf{5}$ and $\mathbf{6}$ by reaction with $\mathrm{BBr}_{3}$ led to $\mathbf{2}$ as $E$ and $Z$ isomers both in racemic mixture. The structure of $\mathbf{2}$ has been characterized by homonuclear $\left({ }^{1} \mathrm{H}\right.$ and $\left.{ }^{13} \mathrm{C}\right)$ and heteronuclear $\left({ }^{1} \mathrm{H}-{ }^{1} \mathrm{H}\right.$ and $\left.{ }^{1} \mathrm{H}_{-}{ }^{13} \mathrm{C}\right) \mathrm{NMR}$ analysis. In particular, NOE experiments have been used to discriminate between the $E$ and $Z$ configurations. In fact, contacts between $\mathrm{H} 4^{\prime}$ and $\mathrm{H} 7$ were observed in the dominant diastereoisomer and contacts 
between $\mathrm{H}^{\prime}$ ' and $\mathrm{HA}$ in the minor one allowing the configuration assignment to $E$ and $Z$ respectively. Moreover, the chemical structures of the chiral intermediates 5, E-6 and Z-6 in racemic form were confirmed by X-ray crystallography (Fig. SI-1) while 2 does not crystallize.

Spectroscopy and photochemistry. We now report on the stationary UV-Vis, photochemical and ECD characterization and on the transient UV-Vis characterization of 2 , while the related computational studies are presented later. An HPLC setup equipped with a semi-preparative column (Phenomenex Lux $5 \mu \mathrm{m}$ Cellulose-1, LC Column 250 x $4.6 \mathrm{~mm}$ ) and applying n-hexane with traces (3\%) of isopropyl acid as mobile phase was used to separate the $E$ and $Z$ diastereomers of 2 in their neutral form. The absorption spectra of the pure diastereomers were then recorded in n-hexane (Fig. 2). The Z-2 band was found to be red-shifted with respect to the $E-\mathbf{2}$ band.

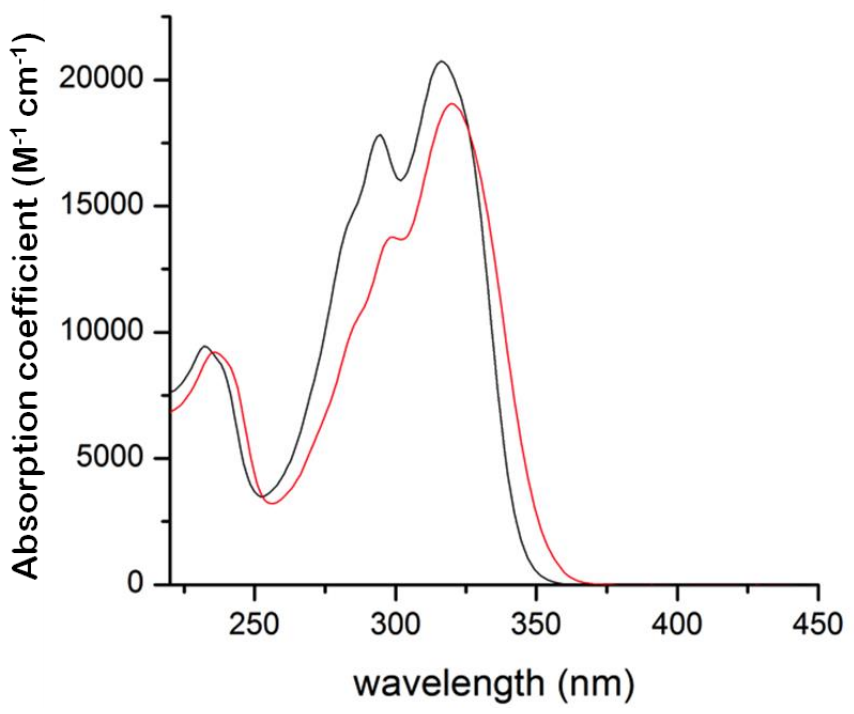

Figure 2. Quantitative absorption spectra of E-2 (black line) and Z-2 (red line) in n-hexane.

Similar to $E-\mathbf{1},{ }^{38}$ the absorption spectrum of $E-\mathbf{2}$ was recorded at $\mathrm{pH}$ values stabilizing the neutral and anionic forms and in different solvents (Fig. 3). The wavelength of the absorption maxima $\left(\lambda_{\max }\right)$ are reported in Table 1. In methanol (Fig. 3, red line) the neutral form of $E-2$ shows two dominant spectral components at 295 and $318 \mathrm{~nm}$ and it is therefore close to those reported for $E-\mathbf{1}$ (297 and $320 \mathrm{~nm}$. Fig. 3 blue line). The two components do not display significant solvatochromic effects $(3-7 \mathrm{~nm}$ difference in $\lambda_{\max }$ ) when replacing hexane with methanol, DMSO, acetonitrile, or water (see SI for 
the absorption spectra in acetonitrile and water). In contrast, the spectrum of the corresponding anionic form in methanol is characterized by a single band $\left(\lambda_{\max }=347 \mathrm{~nm}\right.$. Fig. 3, dashed red line $)$ significantly red-shifted with respect to the neutral form in line with what reported for $E-\mathbf{1}\left(\lambda_{\max }=351\right.$ nm. Fig. 3, blue line). In contrast with its neutral form, the anionic form of $E-\mathbf{2}$ shows a significant solvatochromic effect when passing from methanol to DMSO (36 nm red-shift. Fig. 3) or acetonitrile (15 nm red-shift. Fig. SI-3). The different behaviour of the anionic form may be related to the higher flexibility of its $\pi$-electron density when related to the marked difference in the dipole moment of the two solvents (methanol 1.70, DMSO 3.96).

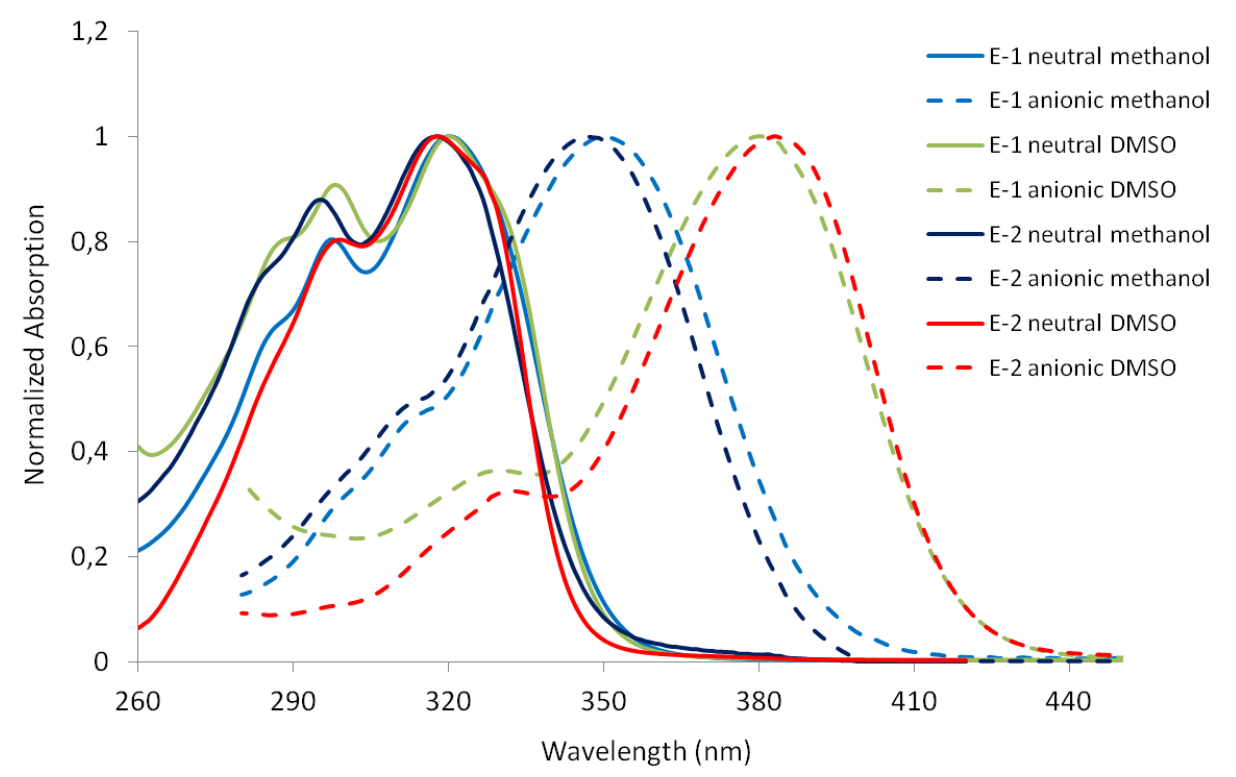

Figure 3. Normalized absorption spectra of the neutral (solid lines) and anionic (dashed lines) form of $E-\mathbf{2}$ in methanol (red) and DMSO (orange) compared to the spectra of the neutral (solid blue line) and anionic (dashed blue line) forms of $E-\mathbf{1}$ in methanol.

Table 1. Absorption maxima ( $\lambda_{\max }$ values) of neutral and anionic $E-2$ in different solvents and PSS composition of neutral and anionic $\mathbf{2}$ in methanol at different irradiation wavelengths. The $\lambda_{\max }$ values for $E \mathbf{- 1}$ are also given.

\begin{tabular}{ccc|c|c}
\hline \multicolumn{2}{c|}{ Absorption maxima in various solvents } & \multicolumn{2}{c}{ Photostationary states in Methanol } \\
\hline Solvent & $E-2$ neutral & $E-2$ anion $^{\mathrm{a}}$ & $\begin{array}{c}\text { Irradiation } \\
\text { wavelength (nm) }\end{array}$ & $\begin{array}{c}E / Z \\
\text { composition } \\
( \pm 0.1)\end{array}$ \\
\hline
\end{tabular}




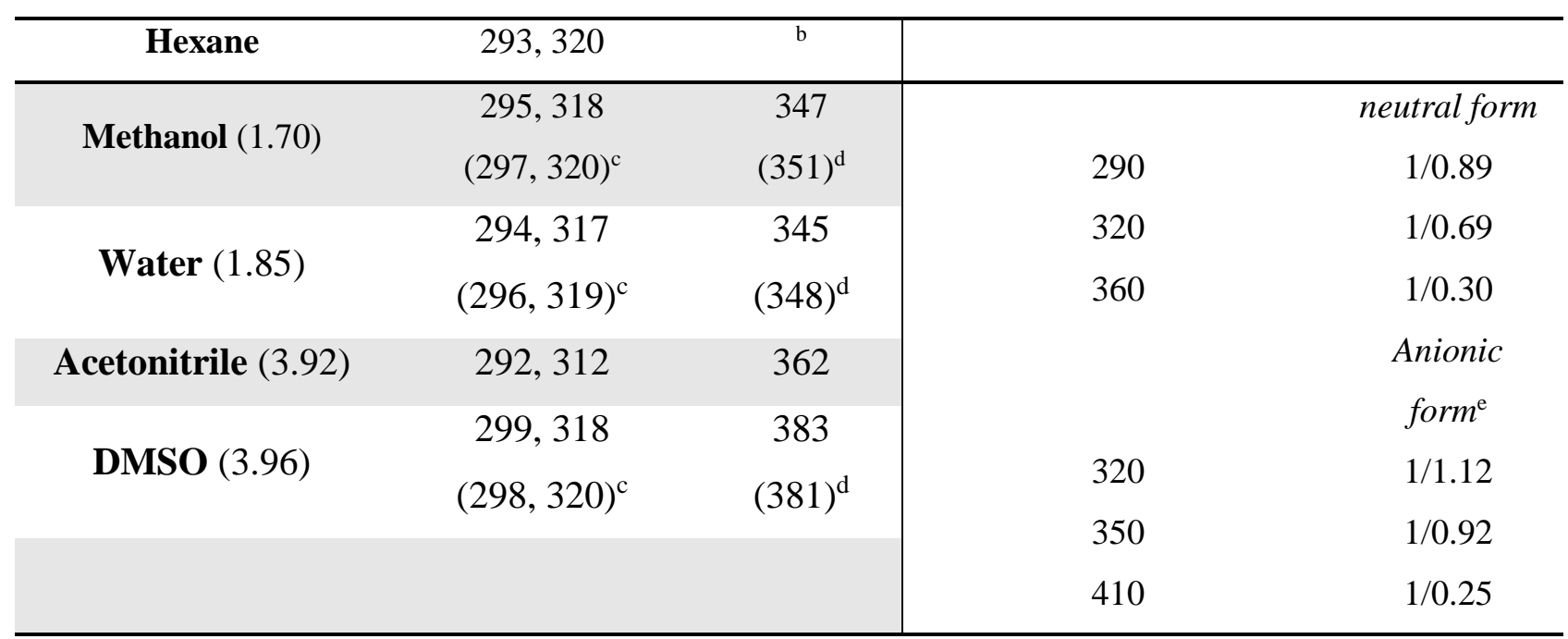

agenerated by addition of $\mathrm{KOH}$ to the neutral solutions; ${ }^{\text {} N o t}$ soluble; ${ }^{\text {cin }}$ brackets the value of compound $E-\mathbf{1}$ in neutral

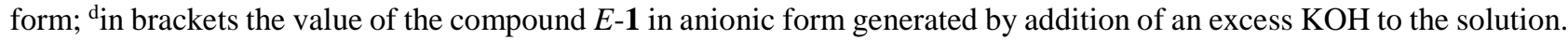

The photostationary state (PSS) of $\mathbf{2}$ was investigated by irradiation in a Pyrex NMR tube at room temperature in methanol- $d_{4}$ using three irradiation wavelengths. The PSS $E / Z$ ratio was determined using ${ }^{1} \mathrm{H}$ NMR spectroscopy (area ratio of the aromatic signals, Table 1 and Fig. SI-4). No significant change in ratio was observed after storing the achieved PSS composition in the dark for a few days suggesting a high energy barrier for thermal $\mathrm{C}=\mathrm{C}$ isomerization at room temperature. Notice that an inversion in the $\mathrm{E} / \mathrm{Z}$ ratio could be only achieved when using the bluer $320 \mathrm{~nm}$ irradiation wavelength on the anionic form.

The absolute photoisomerization quantum yield of the neutral form of $E-2$ was determined to be 0.23 \pm 0.01 in both methanol or n-hexane, as revealed by HPLC analysis and spectrophotometric measurements upon irradiation at $315 \mathrm{~nm}$. Similarly, medium independent photoisomerization quantum yield value of $0.17 \pm 0.01$ was measured by spectrophotometric analysis upon irradiation at $350 \mathrm{~nm}$ for $E-2$ is in the anionic form.

Using the HPLC setup described above, the $E, R-\mathbf{2}, E, S-\mathbf{2}$ and $Z, R-\mathbf{2}$ e $Z, S-\mathbf{2}$ enantiomers were separated by employing a suitable chiral stationary phase. Thus, as reported in Fig. 4, the ECD spectra of each enantiomer could be recorded for both the neutral and anionic forms obtained by using $\mathrm{KOH}$ in methanol solution $(85 \mathrm{mM})$. 

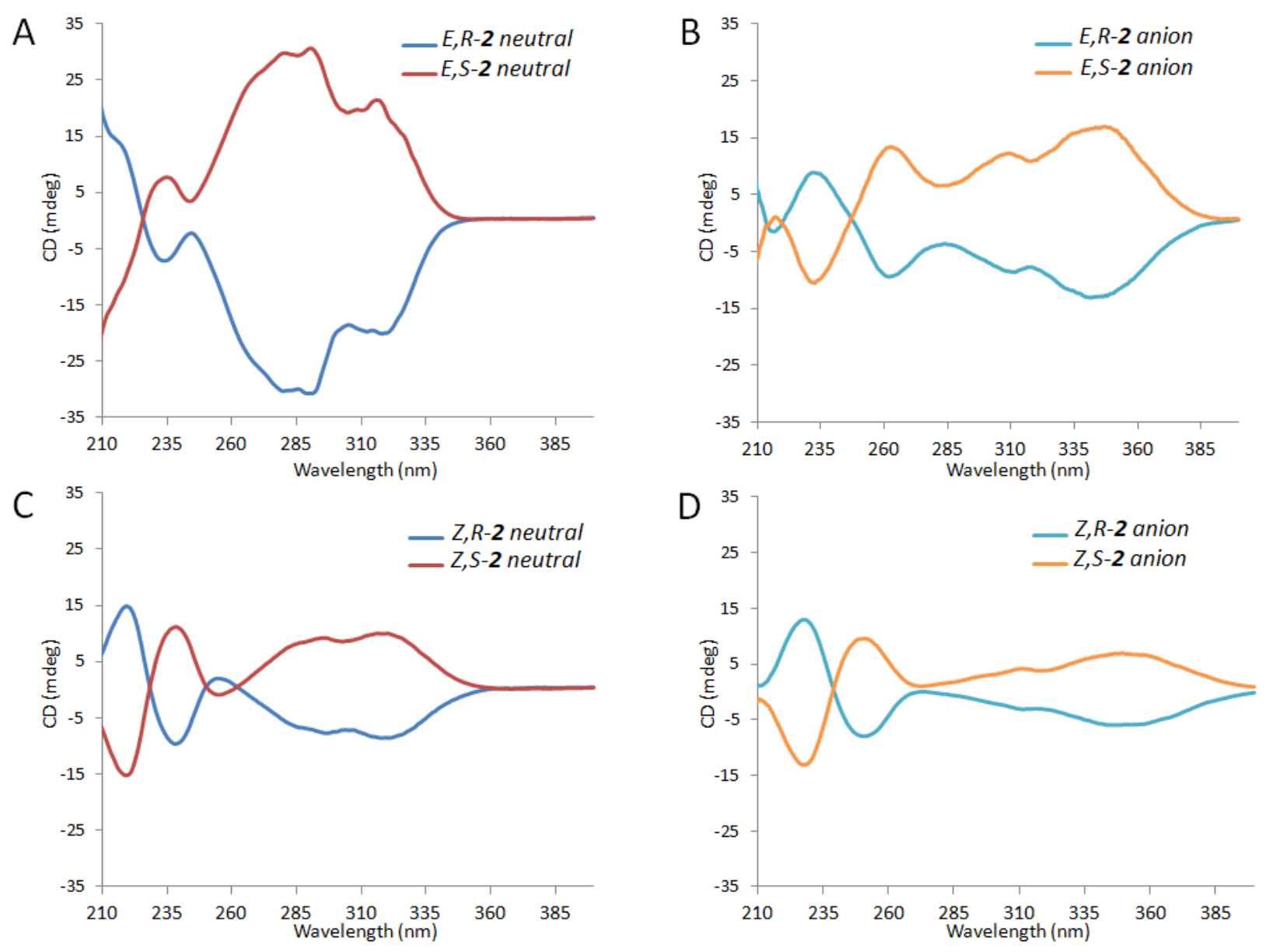

Figure 4. ECD spectra recorded in methanol of the two enantiomers of 2. E,R-2 and E,S-2 in neutral (A) and in anionic (B) form. Z,R-2 and Z,R-2 in neutral (C) and anionic (D) form.

The spectra show distinctive features of the $E$ or $Z$ diastereoisomers which display, as expected, specular spectra for their enantiomers. The HPLC, ECD and computational (see below for the $R$ and $S$ enantiomer assignment) analysis enables the assignment of the spectra of Fig. 4A and 4B to the $E$ diastereoisomers while the spectra of Fig. 4C and 4D are assigned to the $Z$ diastereoisomer. The fact that no racemization was observed upon deprotonation of the neutral forms, enabled to study also the anionic forms. The anion ECD spectra present broader and red-shifted bands. Furthermore, HPLC analysis shows that irradiation does not affect the enantiomeric purity.

Photoisomerization dynamics of the anionic form. The investigation of the light-triggered dynamics of 2 was limited to the biomimetic anionic forms (i.e., this form mimics the GFP fluorophore). Accordingly, a methanol solution of a racemic mixture of the $E-\mathbf{2}$ anion (i.e. generated 
with an excess of $\mathrm{KOH}$ ) was investigated via pump-probe, femtosecond transient absorption spectroscopy (TAS) with a resonant $350 \mathrm{~nm}$ pump pulse. A white-light femtosecond probe pulse was used to measure the time evolution of the absorption spectrum of the generated $\mathrm{S}_{1}$ population with a time resolution of $\sim 60$ fs and a spectral detection window spanning from $350 \mathrm{~nm}$ to $600 \mathrm{~nm}$ (see experimental details and analysis procedures in ref. 38). The resulting spectrum (see e.g. the 0.25 ps spectrum in Fig. 5A) displayed two bands: a positive band peaking around $386 \mathrm{~nm}$ was assigned to

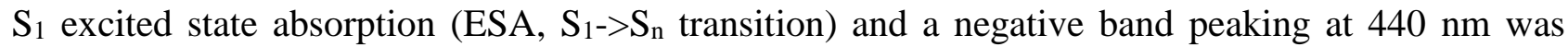
assigned to stimulated emission ( $\mathrm{SE}, \mathrm{S}_{1->}>\mathrm{S}_{0}$ transition).
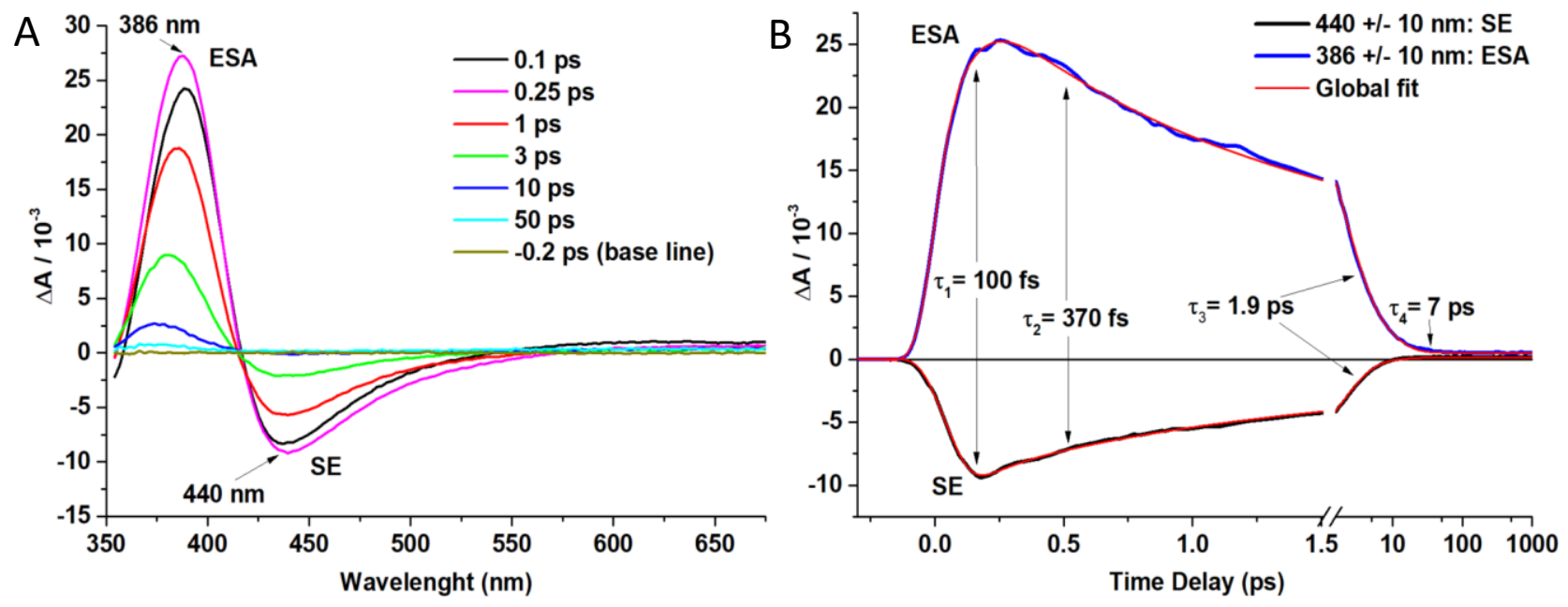

Figure 5. Transient absorption spectra. (A) Selected traces for the $E-2$ anion in methanol upon $350 \mathrm{~nm}$ excitation. (B) Transient absorption kinetics traces as a function of pump-probe time delay (in ps) observed at probing wavelengths of $386 \mathrm{~nm}$. Both kinetic traces overlap with the result of the global, multiexponential fit (red lines). Four time constants $\left(\tau_{1}-\tau_{4}\right)$ displayed on the graph are required for an accurate fit.

Inspection of the spectra reveals the following signatures. As compared to the 0.1 ps spectrum, in the 0.25 ps spectrum the ESA increases and slightly blue shifts while the SE slightly deepens in the range 445-550 $\mathrm{nm}$. This early $\mathrm{S}_{1}$ relaxation is consistent with an overall SE red-shift attributed to vibrational and solvent relaxation. Then, in the 1 ps and 3 ps spectra, both SE and ESA decay significantly. At $10 \mathrm{ps}$, the SE band has completely vanished, and the positive band reaches a maximum at $\lambda \approx 375 \mathrm{~nm}$ (i.e. at blue-shifted values with respect to the $386 \mathrm{~nm}$ maximum of the ESA at $0.25 \mathrm{ps}$ ). Because the SE has completely decayed, we assign the positive band to a photoproduct 
absorption (PA) corresponding to the vibrationally "hot" $Z-2$ in the ground state $\left(\mathrm{S}_{0}\right)$. At $50 \mathrm{ps}$, the photoproduct has thermalized, and the residual signal corresponds to the difference absorption between $E-2$ reactant and $Z-2$ product spectra.

The quantitative analysis of the time evolution of these transient signatures was carried out by global fitting of the entire data set, and illustrated in Fig. 5B at two probing wavelengths. The fitting produced four time-constants. The shortest time-constant ( $\left.\tau_{1}=100 \mathrm{fs}\right)$ characterizes the early spectral relaxation attributed to the initial vibrational and/or solvent relaxations. Two intermediate timeconstants of $\tau_{2}=370$ fs and $\tau_{3}=1.9$ ps were needed to fit the SE and ESA decay and are attributed to $\mathrm{S}_{1}$ population biexponential decay kinetics. A longer time constant $\tau_{4}=7 \mathrm{ps}$ was required to fit further spectral relaxation in the UV range after $S_{1}$ decay and is assigned to thermalization of the hot $S_{0}$ population.

The main result of our TAS study is that two intermediate time-constants $\tau_{2}$ and $\tau_{3}$ describe the $S_{1}$ to $\mathrm{S}_{0}$ decay, with the longest of the two time-constant dominating dominating (70\% of the total $\mathrm{S}_{1}$ decay amplitude monitored at $386 \mathrm{~nm}$, see fig. 5B). Hence an average $\mathrm{S}_{1}$ decay time of $1.4 \mathrm{ps}$ can be inferred. This is a factor 10 times longer than the $S_{1}$ lifetime of the achiral anion $E-1,{ }^{39}$ where the dominating (90\%) component was observed to be $120 \mathrm{fs,} \mathrm{while} \mathrm{a} \mathrm{minor} \mathrm{(10 \% )} \mathrm{second} \mathrm{decay} \mathrm{time} \mathrm{was} \mathrm{found} \mathrm{in}$ the 0.4 to 0.8 ps time range

As anticipated above, one would expect that a rotor embedded in an asymmetric environment rotates faster possibly due to a large initial out-of-plane distortion leading to a double bond pre-twisting in $\mathrm{S}_{0}$ and, in turn, a decrease in $\mathrm{S}_{1}$ lifetime. Such a correlation between an $\mathrm{S}_{0} \mathrm{C}=\mathrm{C}$ pre-twist and a faster $\mathrm{C}=\mathrm{C}$ photoisomerization has been discussed in NAIP systems. ${ }^{35}$ The observed increase in $\mathrm{S}_{1}$ lifetime of $E-2$ with respect to $E-1$ is therefore counterintuitive and calls for a conformational analysis that was carried out at the computational level (see below). Here we observe that the comparison of the crystallographic structures of the asymmetric E-6 intermediate in Fig. SI-1 and of its formally symmetric analogue E-7 (previously reported in ref. 39) suggests that $E-\mathbf{2}$ and $E-\mathbf{1}$ are conformationally different (notice that it has not been possible to obtain suitable crystals for $E-2$ and 
$E$-1). In fact, the removal of the methyl group at $\mathrm{C} 2$ must result in an increased planarity due to a reduced steric hindrance at the indanylidene stator level. As shown in Fig. 6, the structure of E-7 shows that $\mathrm{C} 2$ is lying outside the plane of the aromatic ring of the indanylidene stator. Consequently, the pyrrolidinone rotor places its carbonyl oxygen in the middle of the two methyl groups at $\mathrm{C} 2$ of the stator (torsion angle $\mathrm{C} 1=\mathrm{C} 3^{\prime}-\mathrm{C} 2^{\prime}=\mathrm{O}$ ca. $-13^{\circ}$ ) forcing the rotor $\mathrm{C} 4{ }^{\prime}$ carbon out of the amide bond plane (torsional angle $\mathrm{C}^{\prime}-\mathrm{N} 1-\mathrm{C} 55^{\prime}-\mathrm{C} 4 '$ ca. $6^{\circ}$ ). While a similar $\mathrm{C} 2$ placement is present in $E-6$, the reduced steric hindrance allows the carbonyl oxygen to interact with the hydrogen at $\mathrm{C} 2$ in a $\mathrm{C}=\mathrm{O}---$ H-C- contact (distance of $2.4 \AA$ ) also suggesting formation of an unconventional intramolecular hydrogen bond interaction (torsion angle $\mathrm{C} 1=\mathrm{C} 3^{\prime}-\mathrm{C} 2^{\prime}=\mathrm{O}$ ca. $-5.5^{\circ}$ ) which would further stabilize a planar framework. Notice that all atoms of the pyrrolidone moiety of $E-6$ are located on approximately the same plane (torsion angle $\left.\mathrm{C} 2^{\prime}-\mathrm{N} 1-\mathrm{C} 5^{\prime}-\mathrm{C} 4{ }^{\prime}=-5.0^{\circ}\right)$. Similarly, the pyrrolidinone head of E-6 seems less strained than the pyrrolidinone head of E-7. Assuming that these conformational differences are maintained in $E-\mathbf{1}$ and $E-\mathbf{2}$, the reduced helicity and, thus, C=C pretwist of $E-2$ would be in line with a longer $S_{1}$ lifetime.

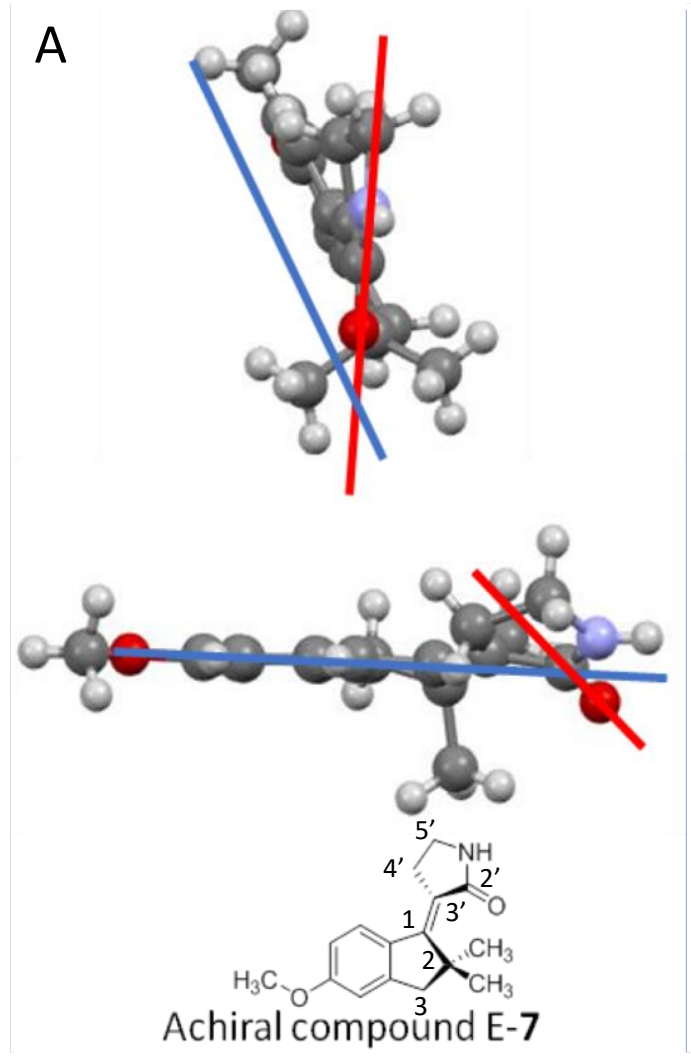

B
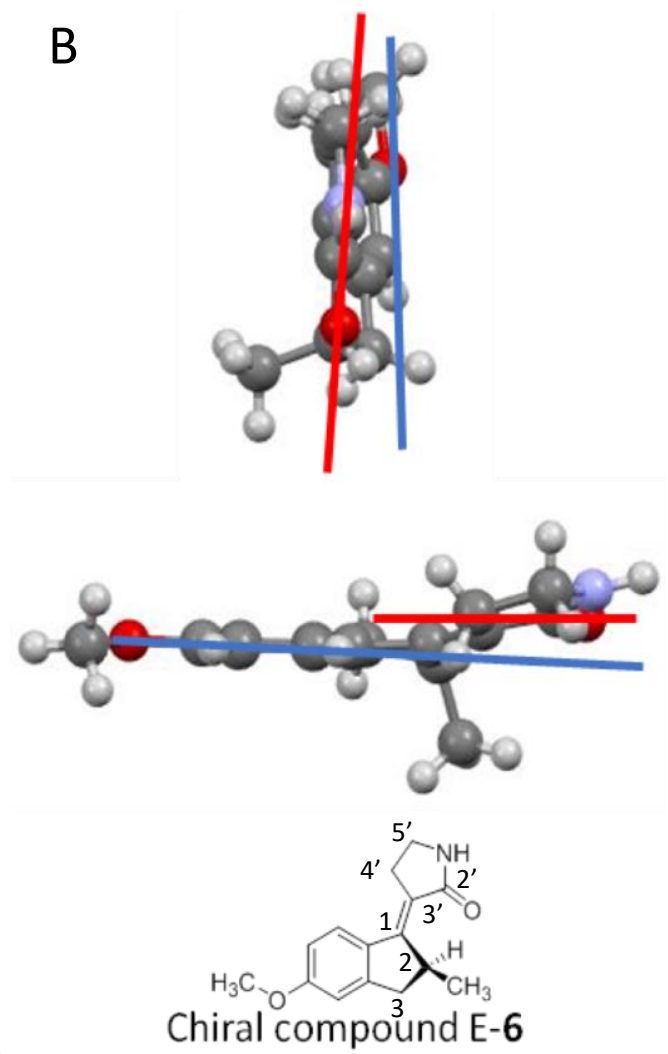

Figure 6. Comparison of X-ray crystallographic structures. (A) achiral E-7 and (B) chiral E-6. 
Conformational analysis in solution and simulation of the stationary and transient ECD spectra. Following the TAS studies above, we carried out two distinct computational investigations focusing, exclusively, on the anionic form of $\mathbf{2}$ and, for comparison, of $\mathbf{1}$. The $\mathrm{S}_{0}$ room-temperature conformational equilibrium (i.e. their Boltzmann distribution) of $E-\mathbf{1}$ and $E-2$ in methanol solution has been simulated using a quantum-mechanics/molecular-mechanics (QM/MM) model where the QM subsystem (the solute) is treated using a multiconfigurational post-HF level of theory (CASPT2//CASSCF/6-31G*) while the MM subsystem (the solvent) is treated using the Amber force field. ${ }^{40}$ Above it has been hypothesized that, counterintuitively, the stereogenic center in E-2 makes it more planar with respect to $E-\mathbf{1}$ due to a reduced steric hindrance. In order to support this hypothesis, we have employed our model to run classical molecular dynamics (MD) at room temperature for both systems. The resulting distributions, represented by a set of 400 uncorrelated geometries and velocities (i.e. MD trajectory snapshots), allow to simulate the $E-\mathbf{1}$ and $E-\mathbf{2}$ absorption bands (see Fig. 7) by computing the corresponding 400 vertical excitation energy values. Therefore, we concluded that the corresponding distribution of geometrical parameters as well as their average values provide information on the solute conformational structures.
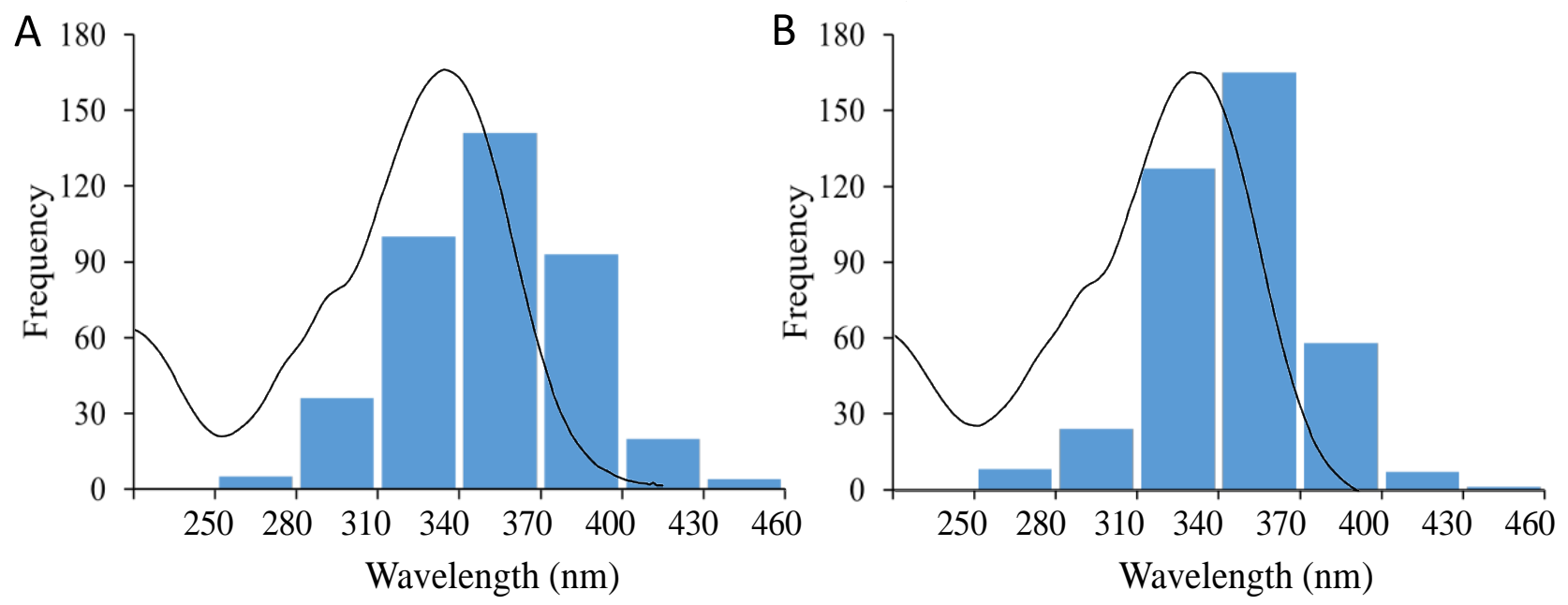

Figure 7. Comparison between experimental (solid lines) and computed (histogram) absorption bands of the anionic forms of the (A) achiral compound $E-\mathbf{1}$ and (B) chiral $E-\mathbf{2}$. 
In fact, encouraged by the fact that the computed $\lambda_{\max }$ values (352 and $346 \mathrm{~nm}$ for $E-\mathbf{1}$ and $E-2$ respectively) are consistent with the observed quantities of Table 1, we have estimated the "planarity" of the $E-\mathbf{1}$ and $E-\mathbf{2}$ anions by looking at the values of a set of dihedrals angles (Fig. 8). These include the $\mathrm{C} 1=\mathrm{C} 3^{\prime}-\mathrm{C} 2{ }^{\prime}=\mathrm{O}$ and $\mathrm{C} 2^{\prime}-\mathrm{N} 1-\mathrm{C}^{\prime}-\mathrm{C} 4^{\prime}$ dihedrals (see above) and the $\mathrm{C} 2^{\prime}-\mathrm{C} 3^{\prime}=\mathrm{C} 1-\mathrm{C} 2$ dihedral describing the reactive double-bond distortion from the point of view of the pyrrolidinone rotor and of the dihedrals C3a-C3-C2-C1, C2-C3-C3a-C7a, and C3-C2-C1=C3' to evaluate the stability of the stator. For $E-\mathbf{1}$ the data show (Fig. 8A and 8C) the expected equilibrium between approximate mirror image structures (i.e. the two enantiomeric conformations) displaying a helical framework both in stator and rotor portions. However, for $E, S-\mathbf{2}$, where the $\mathrm{C} 2$ stereogenic center is present, only C2'N1-C5'-C4' conserve a mirror-image aspect while $\mathrm{C} 1=\mathrm{C} 3{ }^{\prime}-\mathrm{C} 2{ }^{\prime}=\mathrm{O}$ and, most importantly, the other dihedrals show that only one value of the dihedrals is statistically dominant at equilibrium (Fig. 8BD). While this points to the expected loss of one of the two equivalent pseudo-mirror-image conformers, we notice that (i) the distribution become substantially broader and (ii) the chiral $E, S$-2 solute is substantially more planar than the achiral $E-\mathbf{1}$ solute. This increased planarity confirms the hypothesis proposed on the basis of the intermediate X-ray crystallographic structures seen above a and points to dominant E-2 planar conformation brought about by the reduction in steric hindrance. An important comparison is that between the simulated conformational equilibrium and the X-ray crystallographic structures described above. For instance, it has been seen that the experimental structures show $\mathrm{C} 1=\mathrm{C} 3^{\prime}-\mathrm{C} 2^{\prime}=\mathrm{O}$ dihedrals of ca. $-13^{\circ}$ and $-5.5^{\circ}$ for the achiral and chiral precursors respectively. This result seems to be in line with the data in the left panels of Fig. 8A and 8B which refer to $E-\mathbf{1}$ and $E-\mathbf{2}$ respectively. It is, in fact, evident that $E-\mathbf{1}$ displays a broader angle distribution going from $+15^{\circ}$ to $-15^{\circ}$ while $E-2$ displays a narrower distribution centered around a $-5^{\circ}$ angle. Consistently, the double bond torsion described by the $\mathrm{C} 2-\mathrm{C} 1=\mathrm{C} 3$ '-C2' dihedral shows a broader and flat ca. $+9^{\circ}$ to $-9^{\circ}$ distribution for $E-\mathbf{1}$ and a shaper distribution for the chiral compound centered at $3^{\circ}$ for $E-2$. 

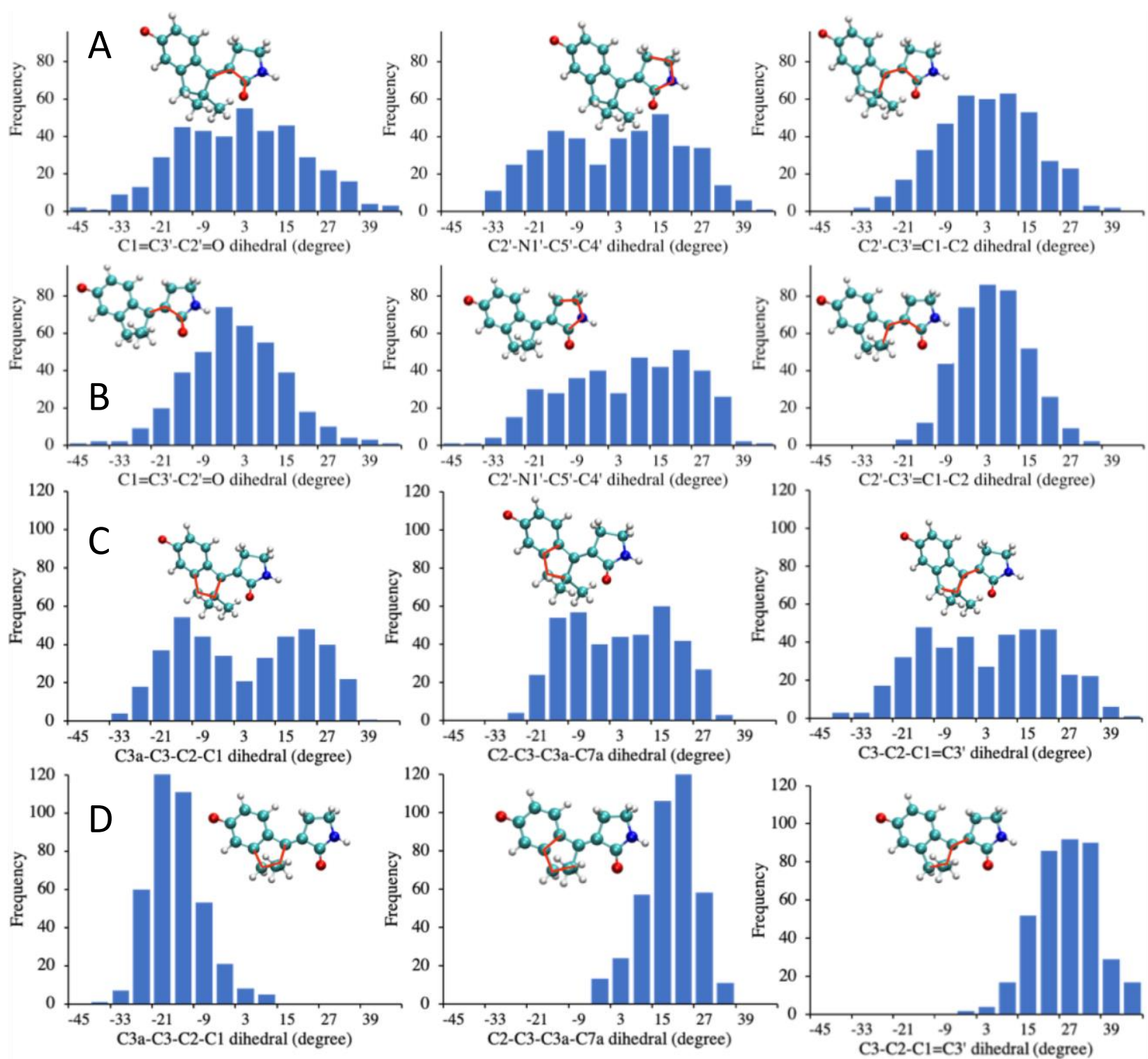

Figure 8. Comparison between the distribution of the dihedral angles describing rotor conformations for the anionic forms of (A) achiral E-1 and (B) chiral E,S-2 and of the dihedrals representing stator conformation of (C) achiral E-1 and (D) chiral E,S-2 in methanol solution. In all cases the dihedrals are indicated by a red segmented line on the corresponding molecular structure representation.

While we continue to focus on the anionic forms of $E, S-\mathbf{2}$ and $Z, S-2$ in methanol solution, a different computational model is employed to enable an ECD study. In fact here we employ a polarizable QM/MM approach, in which the QM subsystem (the solute) is treated at the DFT level of theory (CAM-B3LYP/6-311+G*) and the polarizable Fluctuating Charge (FQ) force field is used for the 
MM subsystem (the solvent). ${ }^{41,42}$ As previously reported, such QM/FQ model is able to reliably simulate solvent effects on chiroptical properties. ${ }^{43,44}$

The QM/FQ ECD spectra were simulated to assign the absolute configurations of the $E$-2 and Z-2 enantiomers based on experimental spectra of Fig. 4B and 4D, respectively. To do so we extracted from the output of the MD simulation discussed above for studying the room-temperature equilibrium, a set of 400 snapshots and computed the corresponding ECD signal. The raw data arising from the sampling are reported on the top panels of Fig. 9A and 9B. For both $E-2$ and Z-2 the stick spectrum shows a high variability in both excitation energy and sign of the rotatory strength. Remarkably, it is found that similar excitations can result in peak intensities of opposite sign which can be attributed to the specific spatial arrangement of the solvent around the solute and on the specific conformation of the latter. The final QM/FQ spectrum is obtained by averaging over the phase-space configurations and it is reported on the bottom panels of Fig. 9A and 9B. The experimental ECD spectra from Fig. 4B and 4D, now assigned to the E,S-2 and Z,S-2 enantiomers of the anionic forms, are reported in the same panels to facilitate comparison. Notice that on this basis we also assign the absolute configuration of the corresponding neutral forms.

The ECD spectrum of the $E, S-2$ anion is characterized by a $(-,+,+,+)$ pattern resulting from a fine balance of sign alternation (see QM/FQ stick spectrum). The same conclusions apply to the Z,S-2 anion (see Fig. 9B). For both diasteroisomers the QM/FQ model correctly predicts the band broadening associated to each band, i.e. the inhomogenous band broadening arising from the sampling of the phase-space via MD. In both cases, the comparison between experimental and QM/FQ spectra shows only small discrepancies in peaks relative intensities, which are probably due to an incorrect description of high-energy excited states provided by the selected DFT level. The agreement between computed and experimental spectra is attributed to the correct description of specific solute-solvent interactions, such as hydrogen bonding (HB) as well as to correctly simulated conformational freedom. In fact, as previously documented, these are appropriately modelled by coupling the polarizable QM/FQ approach with MD runs. ${ }^{45,46}$ 

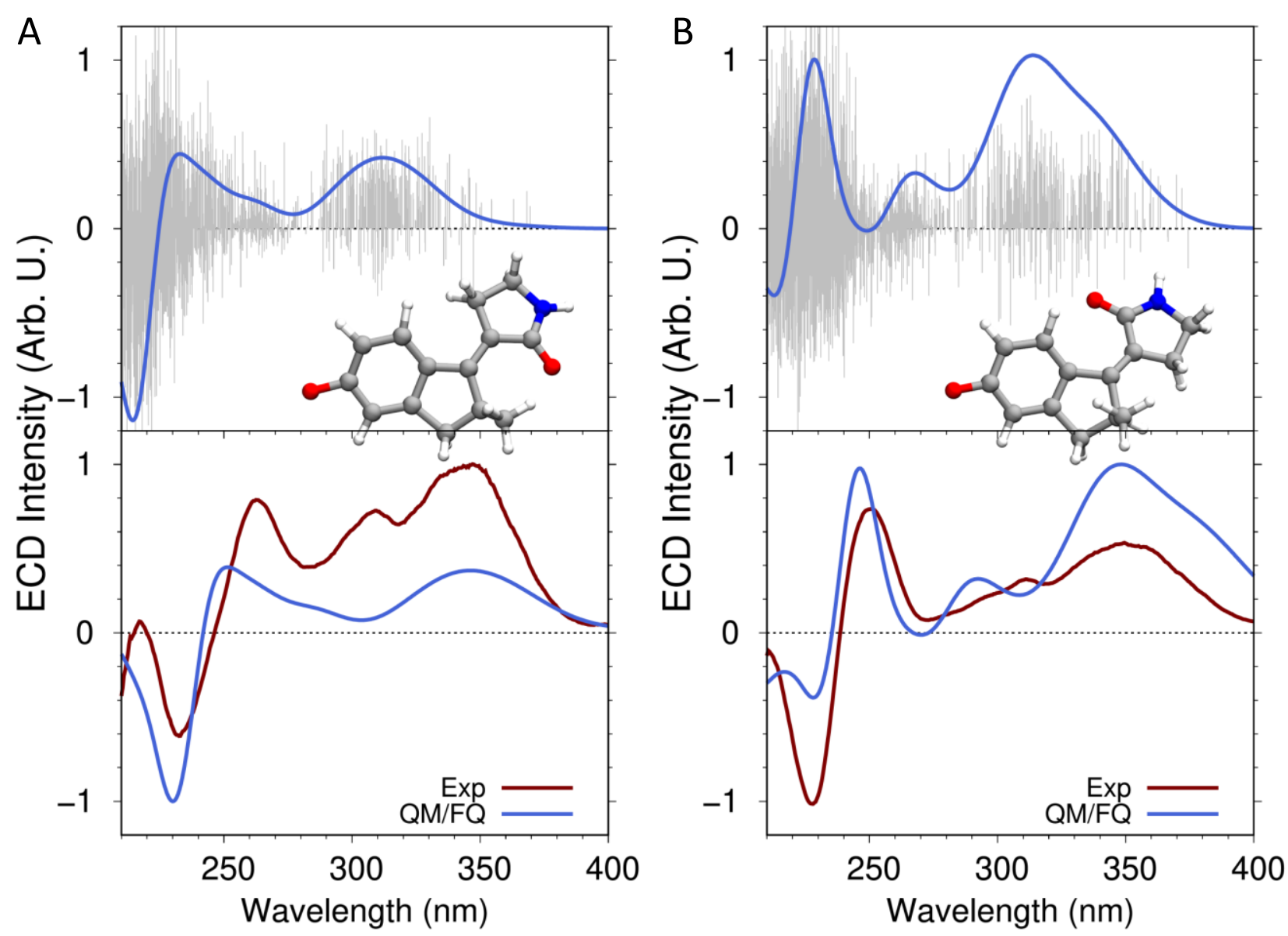

Figure 9. QM/FQ ECD spectra of anionic (A) E,S-2 and (B) Z,S-2 in methanol solution. In both cases raw data are shown as stick spectra (top panels). The convoluted QM/FQ spectra and their comparison with the measured spectrum are given in the bottom panels.

In order to assess the suitability of E,S-2 as a lab model for future time-resolved ECD studies, we investigated the progression of the ECD spectra along the $\mathrm{S}_{0}$ relaxation path populated immediately after $\mathrm{S}_{1}$ decay at a $\mathrm{S}_{1} / \mathrm{S}_{0}$ conical intersection (CoIn) and leading to the Z,S-2 product. For this reason a CoIn has been first optimized at the QM/MM level starting from the already obtained E,S-2 model. At such CoIn the reactive $\mathrm{C}=\mathrm{C}$ bond is approximately $90^{\circ}$ twisted (i.e. it is located half-way along the isomerization coordinate, see Fig. 10A and SI-4B). Accordingly, the QM/MM model defined above was used to (i) locate and optimize the CoIn and (ii) propagate a QM/MM classical trajectory 
starting from the CoIn structure with zero initial velocities. To account for the extremely fast $\mathrm{S}_{0}$ relaxation, the solvent has been kept frozen at the CoIn geometry during the downhill propagation. ECD spectra in methanol were calculated along a collection of trajectory structures describing the $\mathrm{S}_{0}$ relaxation towards the $Z, S-2$ photoproduct. To do so, a QM/FQ model (see above) with the electronic degrees of freedom of the solvent (the FQ charges) adjusted to the transition density was constructed for each path point. Thus, the ECD spectra are computed in a sort of non-equilibrium regime, being the solvent positions frozen, whereas the solute electronic degrees of freedom are left free to re-adjust. Such an assumption is justified by considering the time-scales associated to the different reorganization processes. ${ }^{47}$ The resulting ECD spectral evolution is reported in Fig. 10B in terms of a $2 \mathrm{D}$ plot as a function of the $\mathrm{C} 2{ }^{\prime}-\mathrm{C} 3^{\prime}=\mathrm{C} 1-\mathrm{C} 2$ dihedral angle ( $\delta$, Fig. $\left.10 \mathrm{~A}\right)$. QM/FQ simulated ECD spectra for 10 selected dihedral angles are reported in Fig. 10C. The results show that the spectrum undergoes dramatic changes during the photoisomerization and indicate that such changes should be detectable via time-resolved ECD measurements. In other words, it is concluded that transient ECD spectroscopy with sufficient time resolution is likely to be able to provide information on the photoisomerization of the prepared LDMM possibly resolving the unidirectional rotation direction at the population level.

A
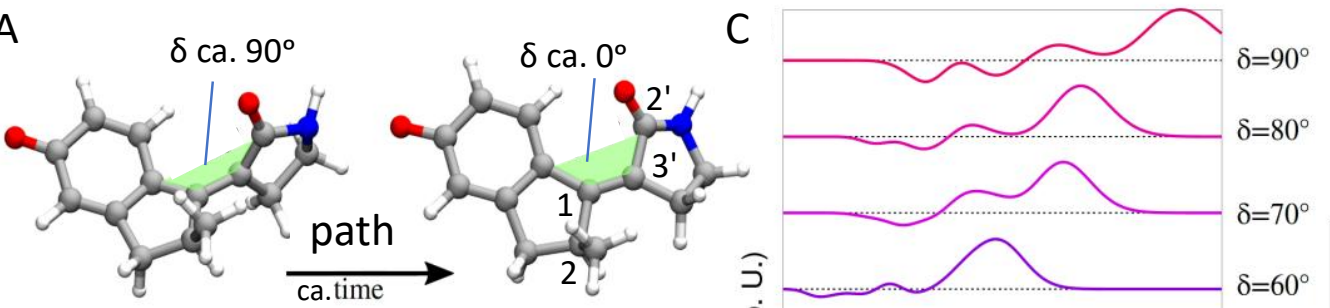

Coln

$Z, S-2$

B
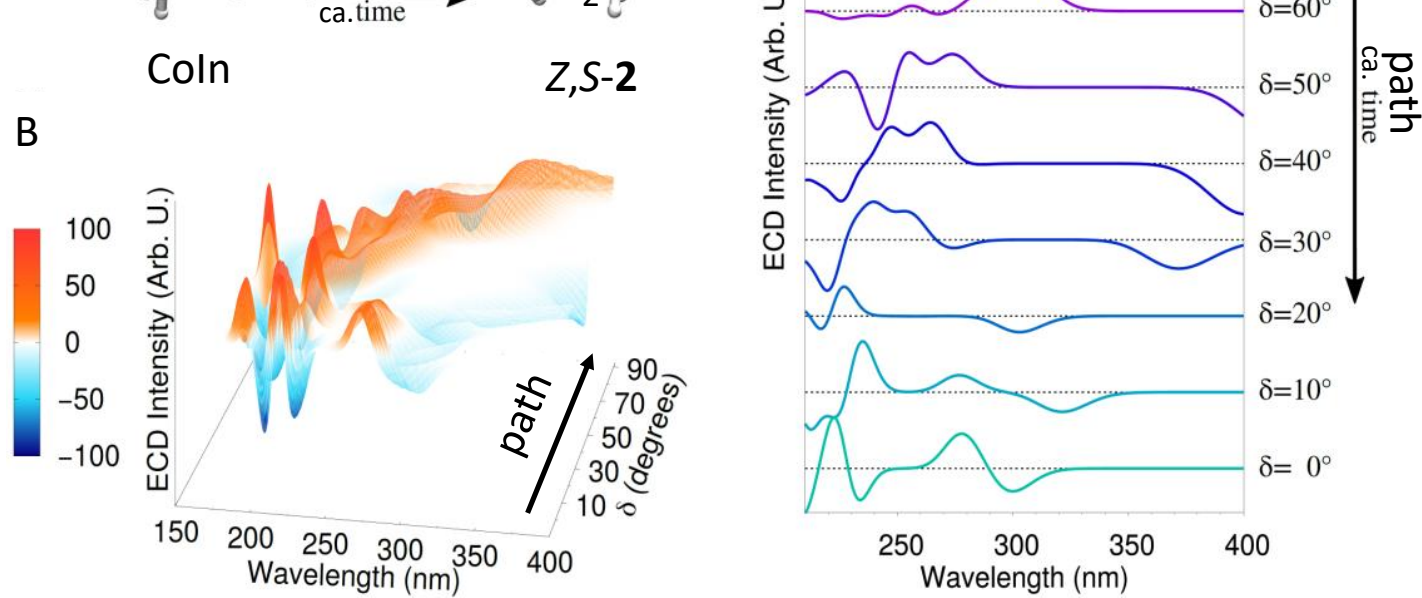
Figure 10. Computed QM/FQ ECD spectra evolution along the $\mathrm{S}_{0}$ relaxation path populated during the $E, S-2$ photoisomerization. (A) Schematic representation of the $S_{0}$ relaxation process in terms of the initial and final dihedral angle $\delta$. (B) Computed QM/FQ ECD spectrum along the relaxation path. (C) Computed QM/FQ ECD spectra during the same process for selected values of $\delta$.

Above we have provided evidence that the asymmetrization of an anionic LDMS generates a prototype LDMM useful for transient ECD studies. In fact, a strong ECD band with good signal-tonoise ratio demonstrates that $\mathbf{2}$ is a plausible model for ECD experiments. Furthermore, the successful comparison between simulated and observed stationary ECD spectra suggests that such experiments could be planned and integrated by QM/MM and QM/FC simulations. On the other hand, TSA studies have also shown that $E-2$ features a substantial decrease in photoisomerization speed relative to its achiral LDMS analogue. Such a decrease (from 0.25 to $1.5 \mathrm{ps}$ in terms of excited state lifetime), is attributed to the planarization of the system framework following the removal of a methyl group in the region connecting stator and rotor. This indicates that the replacement of one of the two methyl substituents of $\mathbf{1}$ with a larger (e.g. an ethyl or, better, isopropyl group), rather than smaller, group would lead to an ideal LDMM with a conserved or increased skeletal out-of-plane deformation (i.e. molecular helicity) and therefore, isomerization speed.

\section{Acknowledgments}

MO is grateful for support from Fondazione Banca d'Italia. The authors are also grateful for a Department of Excellence Grant 2018-2023 funded by the Italian MIUR. We acknowledge support from the Interdisciplinary Thematic Institute QMat, as part of the ITI 2021-2028 program of the University of Strasbourg, CNRS and Inserm, via the IdEx Unistra (ANR 10 IDEX 0002), SFRI STRAT'US (ANR 20 SFRI 0012), EUR QMAT (ANR-17-EURE-0024) and Labex NIE (ANR-11LABX-0058_NIE) projects of the French Investments for the Future Program.

\section{Associated content}


The Supporting Information is available free of charge on the ACS Publications website at DOI:

\section{References}

(1) Mallik, R.; Gross, S. P. Molecular Motors: Strategies to Get Along. Curr. Biol. 2004, 14 (22), R971-R982. https://doi.org/10.1016/j.cub.2004.10.046.

(2) Schliwa, M.; Woehlke, G. Molecular Motors. Nature 2003, 422 (6933), 759-765. https://doi.org/10.1038/nature01601.

(3) Iino, R.; Kinbara, K.; Bryant, Z. Introduction: Molecular Motors. Chem. Rev. 2020, 120 (1), 1-4. https://doi.org/10.1021/acs.chemrev.9b00819.

(4) Chen, J.; Leung, F. K.-C.; Stuart, M. C. A.; Kajitani, T.; Fukushima, T.; van der Giessen, E.; Feringa, B. L. Artificial Muscle-like Function from Hierarchical Supramolecular Assembly of Photoresponsive Molecular Motors. Nat. Chem. 2018, 10 (2), 132-138. https://doi.org/10.1038/nchem.2887.

(5) Feringa, B. L. The Art of Building Small. Chem. Int. 38 (5), 2-7. https://doi.org/https://doi.org/10.1515/ci-2016-0502.

(6) Roke, D.; Wezenberg, S. J.; Feringa, B. L. Molecular Rotary Motors: Unidirectional Motion around Double Bonds. Proc. Natl. Acad. Sci. 2018, 115 (38), 9423-9431. https://doi.org/10.1073/pnas.1712784115.

(7) Kassem, S.; van Leeuwen, T.; Lubbe, A. S.; Wilson, M. R.; Feringa, B. L.; Leigh, D. A. Artificial Molecular Motors. Chem. Soc. Rev. 2017, 46 (9), 2592-2621. https://doi.org/10.1039/C7CS00245A.

(8) Zhang, Q.; Qu, D.-H.; Tian, H.; Feringa, B. L. Bottom-Up: Can Supramolecular Tools Deliver Responsiveness from Molecular Motors to Macroscopic Materials? Matter 2020, 3 (2), 355-370. https://doi.org/https://doi.org/10.1016/j.matt.2020.05.014.

(9) Le Poul, N.; Colasson, B. Electrochemically and Chemically Induced Redox Processes in Molecular Machines. ChemElectroChem 2015, 2 (4), 475-496. 
https://doi.org/10.1002/celc.201402399.

(10) Baroncini, M.; Silvi, S.; Credi, A. Photo- and Redox-Driven Artificial Molecular Motors. Chem. Rev. 2020, 120 (1), 200-268. https://doi.org/10.1021/acs.chemrev.9b00291.

(11) Koumura, N.; Geertsema, E. M.; van Gelder, M. B.; Meetsma, A.; Feringa, B. L. Second Generation Light-Driven Molecular Motors. Unidirectional Rotation Controlled by a Single Stereogenic Center with Near-Perfect Photoequilibria and Acceleration of the Speed of Rotation by Structural Modification. J. Am. Chem. Soc. 2002, 124 (18), 5037-5051. https://doi.org/10.1021/ja012499i.

(12) Vicario, J.; Meetsma, A.; Feringa, B. L. Controlling the Speed of Rotation in Molecular Motors. Dramatic Acceleration of the Rotary Motion by Structural Modification. Chem. Commun. 2005, No. 47, 5910-5912. https://doi.org/10.1039/B507264F.

(13) Filatov, M.; Paolino, M.; Min, S. K.; Kim, K. S. Fulgides as Light-Driven Molecular Rotary Motors: Computational Design of a Prototype Compound. J. Phys. Chem. Lett. 2018, 9 (17), 4995-5001. https://doi.org/10.1021/acs.jpclett.8b02268.

(14) Filatov, M.; Paolino, M.; Min, S. K.; Choi, C. H. Design and Photoisomerization Dynamics of a New Family of Synthetic 2-Stroke Light Driven Molecular Rotary Motors. Chem. Commun. 2019, 55 (36), 5247-5250. https://doi.org/10.1039/C9CC01955C.

(15) Roke, D.; Sen, M.; Danowski, W.; Wezenberg, S. J.; Feringa, B. L. Visible-Light-Driven Tunable Molecular Motors Based on Oxindole. J. Am. Chem. Soc. 2019, 141 (18), 76227627. https://doi.org/10.1021/jacs.9b03237.

(16) Feringa, B. L. The Art of Building Small: From Molecular Switches to Molecular Motors. J. Org. Chem. 2007, 72 (18), 6635-6652. https://doi.org/10.1021/jo070394d.

(17) Chen, J.; Vachon, J.; Feringa, B. L. Design, Synthesis, and Isomerization Studies of LightDriven Molecular Motors for Single Molecular Imaging. J. Org. Chem. 2018, 83 (11), 60256034. https://doi.org/10.1021/acs.joc.8b00654.

(18) Chen, J.; Chen, K.-Y.; Carroll, G. T.; Feringa, B. L. Facile Assembly of Light-Driven 
Molecular Motors onto a Solid Surface. Chem. Commun. 2014, 50 (84), 12641-12644. https://doi.org/10.1039/C4CC04440A.

(19) van Leeuwen, T.; Lubbe, A. S.; Štacko, P.; Wezenberg, S. J.; Feringa, B. L. Dynamic Control of Function by Light-Driven Molecular Motors. Nat. Rev. Chem. 2017, 1 (12), 96. https://doi.org/10.1038/s41570-017-0096.

(20) Zhao, D.; van Leeuwen, T.; Cheng, J.; Feringa, B. L. Dynamic Control of Chirality and SelfAssembly of Double-Stranded Helicates with Light. Nat. Chem. 2017, 9 (3), 250-256. https://doi.org/10.1038/nchem.2668.

(21) Schoenlein, R. W.; Peteanu, L. A.; Mathies, R. A.; Shank, C. V. The first step in vision: femtosecond isomerization of rhodopsin. Science 1991, 254, 412-415. https://doi.org/10.1126/science.1925597

(22) Meyer-Ilse, J.; Akimov, D.; Dietzek, B. Recent Advances in Ultrafast Time-Resolved Chirality Measurements: Perspective and Outlook. Laser Photon. Rev. 2013, 7 (4), 495-505. https://doi.org/10.1002/lpor.201200065.

(23) Auvray, F.; Dennetiere, D.; Giuliani, A.; Jamme, F.; Wien, F.; Nay, B.; Zirah, S.; Polack, F.; Menneglier, C.; Lagarde, B.; et al. Time Resolved Transient Circular Dichroism Spectroscopy Using Synchrotron Natural Polarization. Struct. Dyn. 2019, 6 (5), 54307. https://doi.org/10.1063/1.5120346.

(24) Steinbacher, A.; Hildenbrand, H.; Kramer, C.; Schäferling, M.; Giessen, H.; Brixner, T. Chirality-Sensitive Ultrafast Spectroscopy. In International Conference on Ultrafast Phenomena; Optical Society of America, 2016; p UM3A.1. https://doi.org/10.1364/UP.2016.UM3A.1.

(25) Bonmarin, M.; Helbing, J. Picosecond Time-Resolved Vibrational Circular Dichroism Spectroscopy. In Ultrafast Phenomena XVI; Corkum, P., Silvestri, S., Nelson, K. A., Riedle, E., Schoenlein, R. W., Eds.; Springer Berlin Heidelberg: Berlin, Heidelberg, 2009; pp 391393. 
(26) Bonmarin, M.; Helbing, J. A Picosecond Time-Resolved Vibrational Circular Dichroism Spectrometer. Opt. Lett. 2008, 33 (18), 2086-2088. https://doi.org/10.1364/OL.33.002086.

(27) Zanirato, V.; Pollini, G. P.; De Risi, C.; Valente, F.; Melloni, A.; Fusi, S.; Barbetti, J.; Olivucci, M. Synthesis of Biomimetic Light-Driven Molecular Switches via a Cyclopropyl Ring-Opening/Nitrilium Ion Ring-Closing Tandem Reaction. Tetrahedron 2007, 63, 49754982. https://doi.org/10.1016/j.tet.2007.03.141.

(28) Léonard, J.; Schapiro, I.; Briand, J.; Fusi, S.; Paccani, R. R.; Olivucci, M.; Haacke, S. Mechanistic Origin of the Vibrational Coherence Accompanying the Photoreaction of Biomimetic Molecular Switches. Chem. A Eur. J. A Eur. J. 2012, 18, 15296-15304. https://doi.org/10.1002/chem.201201430.

(29) Dunkelberger, A. D.; Kieda, R. D.; Shin, J. Y.; Rossi Paccani, R.; Fusi, S.; Olivucci, M.; Fleming Crim, F. Photoisomerization and Relaxation Dynamics of a Structurally Modified Biomimetic Photoswitch. J. Phys. Chem. A 2012, 116 (14), 3527-3533. https://doi.org/10.1021/jp300153a.

(30) Lumento, F.; Zanirato, V.; Fusi, S.; Busi, E.; Latterini, L.; Elisei, F.; Sinicropi, A.; Andruniów, T.; Ferré, N.; Basosi, R.; et al. Quantum Chemical Modeling and Preparation of a Biomimetic Photochemical Switch. Angew. Chemie Int. Ed. 2007, 46 (3), 414-420.

(31) Sinicropi, A.; Martin, E.; Ryazantsev, M.; Helbing, J.; Briand, J.; Sharma, D.; Léonard, J.; Haacke, S.; Cannizzo, A.; Chergui, M.; et al. An Artificial Molecular Switch That Mimics the Visual Pigment and Completes Its Photocycle in Picoseconds. Proc. Natl. Acad. Sci. U. S. A. 2008, 105 (46), 17642-17647.

(32) Briand, J.; Braem, O.; Rehault, J.; Leonard, J.; Cannizzo, A.; Chergui, M.; Zanirato, V.; Olivucci, M.; Helbing, J.; Haacke, S. Coherent Ultrafast Torsional Motion and Isomerization of a Biomimetic Dipolar Photoswitch. Phys. Chem. Chem. Phys. 2010, 12 (13), 3178-3187.

(33) Léonard, J.; Briand, J.; Fusi, S.; Zanirato, V.; Olivucci, M.; Haacke, S. Isomer-Dependent Vibrational Coherence in Ultrafast Photoisomerization. New J. Phys. 2013, 15, 105022. 
https://doi.org/10.1088/1367-2630/15/10/105022.

(34) Pagano, K.; Paolino, M.; Fusi, S.; Zanirato, V.; Trapella, C.; Giuliani, G.; Cappelli, A.; Zanzoni, S.; Molinari, H.; Ragona, L.; et al. Bile Acid Binding Protein Functionalization Leads to a Fully Synthetic Rhodopsin Mimic. J. Phys. Chem. Lett. 2019, 10 (9), 2235-2243. https://doi.org/10.1021/acs.jpclett.9b00210.

(35) Gueye, M.; Manathunga, M.; Agathangelou, D.; Orozco, Y.; Paolino, M.; Fusi, S.; Haacke, S.; Olivucci, M.; Léonard, J. Engineering the Vibrational Coherence of Vision into a Synthetic Molecular Device. Nat. Commun. 2018, 9 (1), 313. https://doi.org/10.1038/s41467017-02668-w.

(36) Gueye, M.; Paolino, M.; Gindensperger, E.; Haacke, S.; Olivucci, M.; Léonard, J. Vibrational Coherence and Quantum Yield of Retinal-Chromophore-Inspired Molecular Switches. Faraday Discuss. 2020,221, 299-321. https://doi.org/10.1039/C9FD00062C.

(37) Schapiro, I.; Gueye, M.; Paolino, M.; Fusi, S.; Marchand, G.; Haacke, S.; Martin, M. E.; Huntress, M.; Vysotskiy, V. P.; Veryazov, V.; et al. Synthesis, Spectroscopy and QM/MM Simulations of a Biomimetic Ultrafast Light-Driven Molecular Motor. Photochem. Photobiol. Sci. 2019, 18 (9), 2259-2269. https://doi.org/10.1039/C9PP00223E.

(38) Marchand, G.; Eng, J.; Schapiro, I.; Valentini, A.; Frutos, L. M.; Pieri, E.; Olivucci, M.; Léonard, J.; Gindensperger, E. Directionality of Double-Bond Photoisomerization Dynamics Induced by a Single Stereogenic Center. J. Phys. Chem. Lett. 2015, No. 1, 599-604. https://doi.org/10.1021/jz502644h.

(39) Paolino, M.; Gueye, M.; Pieri, E.; Manathunga, M.; Fusi, S.; Cappelli, A.; Latterini, L.; Pannacci, D.; Filatov, M.; Léonard, J.; et al. Design, Synthesis, and Dynamics of a Green Fluorescent Protein Fluorophore Mimic with an Ultrafast Switching Function. J. Am. Chem. Soc. 2016, 138 (31), 9807-9825. https://doi.org/10.1021/jacs.5b10812.

(40) Cornell, W. D.; Cieplak, P.; Bayly, C. I.; Gould, I. R.; Merz, K. M.; Ferguson, D. M.; Spellmeyer, D. C.; Fox, T.; Caldwell, J. W.; Kollman, P. A. A Second Generation Force 
Field for the Simulation of Proteins, Nucleic Acids, and Organic Molecules. J. Am. Chem. Soc. 1995, 117 (19), 5179-5197. https://doi.org/10.1021/ja00124a002.

(41) Lipparini, F.; Cappelli, C.; Barone, V. Linear Response Theory and Electronic Transition Energies for a Fully Polarizable QM/Classical Hamiltonian. J. Chem. Theory Comput. 2012, 8 (11), 4153-4165. https://doi.org/10.1021/ct3005062.

(42) Cappelli, C. Integrated QM/Polarizable MM/Continuum Approaches to Model Chiroptical Properties of Strongly Interacting Solute-Solvent Systems. Int. J. Quantum Chem. 2016, 116 (21), 1532-1542. https://doi.org/https://doi.org/10.1002/qua.25199.

(43) Giovannini, T.; Egidi, F.; Cappelli, C. Molecular Spectroscopy of Aqueous Solutions: A Theoretical Perspective. Chem. Soc. Rev. 2020, 49 (16), 5664-5677. https://doi.org/10.1039/C9CS00464E.

(44) Giovannini, T.; Egidi, F.; Cappelli, C. Theory and Algorithms for Chiroptical Properties and Spectroscopies of Aqueous Systems. Phys. Chem. Chem. Phys. 2020, 22 (40), 22864-22879. https://doi.org/10.1039/D0CP04027D.

(45) Giovannini, T.; Olszòwka, M.; Cappelli, C. Effective Fully Polarizable QM/MM Approach To Model Vibrational Circular Dichroism Spectra of Systems in Aqueous Solution. J. Chem. Theory Comput. 2016, 12 (11), 5483-5492. https://doi.org/10.1021/acs.jctc.6b00768.

(46) Giovannini, T.; Del Frate, G.; Lafiosca, P.; Cappelli, C. Effective Computational Route towards Vibrational Optical Activity Spectra of Chiral Molecules in Aqueous Solution. Phys. Chem. Chem. Phys. 2018, 20 (14), 9181-9197. https://doi.org/10.1039/C8CP00487K.

(47) Mennucci, B.; Cammi, R.; Tomasi, J. Excited States and Solvatochromic Shifts within a Nonequilibrium Solvation Approach: A New Formulation of the Integral Equation Formalism Method at the Self-Consistent Field, Configuration Interaction, and Multiconfiguration Self-Consistent Field Level. J. Chem. Phys. 1998, 109 (7), 2798-2807. https://doi.org/10.1063/1.476878. 